\title{
A Sieve Bootstrap for the Test of a Unit Root ${ }^{1}$
}

\author{
Yoosoon Chang \\ Department of Economics \\ Rice University \\ and \\ Joon Y. Park \\ School of Economics \\ Seoul National University
}

\begin{abstract}
In this paper, we consider a sieve bootstrap for the test of a unit root in models driven by general linear processes. The given model is first approximated by a finite autoregressive integrated process of order increasing with the sample size, and then the method of bootstrap is applied for the approximated autoregression to obtain the critical values for the usual unit root tests. The resulting tests, which may simply be viewed as the bootstrapped versions of Augmented-Dickey-Fuller (ADF) unit root tests by Said and Dickey (1984), are shown to be consistent under very general conditions. The asymptotic validity of the bootstrap ADF unit root tests is thus established. Our conditions are significantly weaker than those used by Said and Dickey. Simulations show that bootstrap provides substantial improvements on finite sample sizes of the tests.
\end{abstract}

This version: February, 2001

Key words and phrases: Sieve bootstrap, Unit roots, ADF tests, linear process, autoregressive approximation.

\footnotetext{
${ }^{1}$ The authors thank the Editor and an anonymous referee for comments and suggestions. We are also grateful to Don Andrews and Bill Brown for helpful discussions. Park thanks the Department of Economics at Rice University, where he is an Adjunct Professor, for its continuing hospitality and secretarial support. This research is supported by Rice University through CSIV fund, and by the Grant for the Reform of University Education under the Brain Korea 21 Project of SNU 2000. Correspondence address to: Yoosoon Chang, Department of Economics - MS 22, Rice University, 6100 Main Street, Houston, TX 77005-1892, Tel: 713-348-2796, Fax: 713-348-5278, Email: yoosoon@rice.edu.
} 


\section{Introduction}

The unit root hypothesis has drawn much attention for the past two decades especially in economics and other related fields. The hypothesis has an important implication on, in particular, whether or not the shocks to an economic system have a permanent effect on the future path of the economy. Naturally, the research on the subject has been extremely active in both theoretical and empirical domains. Various unit root tests have been proposed and applied to the real data. Although there are some disagreements over how strong and far-reaching is the empirical evidence for the unit root, it seems to be widely agreed that many of important economic and financial time series display unit root characteristics.

For the test of a unit root in a parametric framework, the tests by Dickey and Fuller $(1979,1981)$ are most commonly used. They are based on finite-order autoregressions, the orders of which are assumed to be known. In general, however, it is considered to be undesirable to test for the unit root within a specific parametric family, since if misspecified it could lead us to incorrect inference. There are two approaches to testing for a unit root nonparametrically. The one by Phillips (1987) and Phillips and Perron (1988) relies on the nonparametric modifications of DF (DickeyFuller) tests. The other is to base the tests on autoregressions augmented with lagged differences. Indeed, Said and Dickey (1984) have shown that such ADF (Augmented Dickey-Fuller) tests are valid for all finite ARMA processes of unknown order, if we increase the number of included lagged differences appropriately as the sample size gets large.

The tests by Said-Dickey and Phillips-Perron are often preferred to the DickeyFuller tests in practical applications, since they do not require any particular parametric specification and yet are applicable for a wide class of unit root models. However, the tests are known to have considerable size distortions in finite samples. It has indeed been found by several authors that they may have serious size distortions, especially when the model has moving average components. See Leybourne and Newbold (1999) and the references cited there. Therefore, it seems natural to investigate whether the bootstrap method can improve their finite sample performance.

In this paper, we look at the sieve bootstrap for the unit root tests when the underlying model is driven by general linear processes. The sieve bootstrap approximates the general linear process by a finite autoregressive process of order increasing with the sample size, and resampling from the approximated autoregressions. It is called as such by Bühlmann (1997), since the method is based on an approximation of an infinite dimensional and nonparametric model by a sequence of finite dimensional parametric models. Clearly, it is the most natural bootstrap procedure for the tests. The sieve bootstrap has been studied by Kreiss (1992), Bühlmann (1997) and Bickel and Bühlmann (1999). It has now become a standard tool for the bootstrap from time series, together with the block bootstrap by Künsch (1989).

The bootstrap for the unit root models have previously been studied by several authors. Basawa et al. (1991a, 1991b) consider the bootstrap procedure for the firstorder autoregressive unit root models. They have shown, in particular, that the unit root must be imposed for the generation of bootstrap samples to get consistency for 
the bootstrap unit root tests. Ferretti and Romo (1996) also establish consistency of the tests based on bootstrap critical values for first-order autoregressive models. See Datta (1996) for the subsampling procedure in estimating the first-order autoregressive unit root models.

The rest of the paper is organized as follows. Section 2 introduces ADF tests and present their asymptotic theories. In Section 3, the sieve bootstrap for ADF tests are considered, and the asymptotics for the sieve bootstrap ADF tests are developed. In Section 4, various issues arising in practical implementation of the sieve bootstrap are addressed along with the simulation results for the finite sample performance of the sieve bootstrap ADF tests. Section 5 concludes, and mathematical proofs are collected in Section 6.

\section{ADF Tests and Their Limiting Distributions}

In this section, we introduce ADF tests and their asymptotic distributions. We let the time series $\left(y_{t}\right)$ be given by

$$
y_{t}=\alpha y_{t-1}+u_{t}
$$

with $\left(u_{t}\right)$ generated as

$$
u_{t}=\pi(L) \varepsilon_{t}
$$

where $L$ is the usual lag operator and $\pi(z)=\sum_{k=0}^{\infty} \pi_{k} z^{k}$. The test of the unit root null hypothesis $\alpha=1$ will be considered for $\left(y_{t}\right)$ given as in (1), against the alternative of stationarity $|\alpha|<1 .^{2}$ To implement the test, the infinite moving average process $\left(u_{t}\right)$ will be approximated by a finite autoregression, i.e., $u_{t}=\alpha_{1} u_{t-1}+\cdots+\alpha_{p} u_{t-p}+\varepsilon_{p, t}$, the order $p$ of which is assumed to increase as the sample size $n$ grows. We write $p_{n}$ instead of $p$ in what follows, when it needs to be emphasized that $p$ is a function of $n$.

We make the following assumptions.

Assumption A1 Let $\left(\varepsilon_{t}, \mathcal{F}_{t}\right)$ be a martingale difference sequence, with some filtration $\left(\mathcal{F}_{t}\right)$, such that $\mathbf{E}\left(\varepsilon_{t}^{2} \mid \mathcal{F}_{t-1}\right)=\sigma^{2}$ and $\mathbf{E}\left|\varepsilon_{t}\right|^{r}<K$ for $r \geq 4$ and some constant $K>0$.

Assumption A2 Let $\pi(z) \neq 0$ for all $|z| \leq 1$, and $\sum_{k=0}^{\infty}|k|^{s}\left|\pi_{k}\right|<\infty$ for some $s \geq 1$.

Assumption A3 Let $p_{n} \rightarrow \infty$ and $p_{n}=o\left((n \log n)^{1 / 2}\right)$ as $n \rightarrow \infty$.

Our specification in (2) with the conditions in Assumptions A1 and A2 allows $\left(u_{t}\right)$ to be generated by a quite general linear process. Under Assumptions A1 and A2, in particular, $\left(u_{t}\right)$ becomes a weakly stationary process with uniformly bounded

\footnotetext{
${ }^{2}$ The initial value $y_{0}$ of $\left(y_{t}\right)$ does not affect our subsequent asymptotics, and therefore, we let $y_{0}$ be any random variable which is stochastically bounded.
} 
fourth moment. The conditions in Assumptions A1 - A3 are significantly weaker than the ones used by Said and Dickey (1984). They assume that the innovations $\left(\varepsilon_{t}\right)$ are iid. Moreover, they only consider $\left(u_{t}\right)$ generated by a finite order ARMA process and thus effectively look at the case where $\left(\pi_{k}\right)$ decays geometrically. Their assumption correponds to $s=\infty$ in our Assumption A2. Also, they assume $p_{n}=c n^{\kappa}$ for $0<\kappa \leq 1 / 3$. Therefore, for instance, the logarithmic rate for $p_{n}$ is not allowed in their result.

The test of the unit root hypothesis for the time series $\left(y_{t}\right)$ given by (1) and (2) can now be based on the regression

$$
y_{t}=\alpha y_{t-1}+\sum_{k=1}^{p} \alpha_{k} \triangle y_{t-k}+\varepsilon_{p, t}
$$

Note that under the null hypothesis we have $\alpha=1$ and $\triangle y_{t}=u_{t}$. To introduce the test statistics, we define

$$
x_{p, t}=\left(\triangle y_{t-1}, \ldots, \triangle y_{t-p}\right)^{\prime}
$$

and subsequently let

$$
\begin{aligned}
A_{n} & =\sum_{t=1}^{n} y_{t-1} \varepsilon_{p, t}-\left(\sum_{t=1}^{n} y_{t-1} x_{p, t}^{\prime}\right)\left(\sum_{t=1}^{n} x_{p, t} x_{p, t}^{\prime}\right)^{-1}\left(\sum_{t=1}^{n} x_{p, t} \varepsilon_{p, t}\right) \\
B_{n} & =\sum_{t=1}^{n} y_{t-1}^{2}-\left(\sum_{t=1}^{n} y_{t-1} x_{p, t}^{\prime}\right)\left(\sum_{t=1}^{n} x_{p, t} x_{p, t}^{\prime}\right)^{-1}\left(\sum_{t=1}^{n} x_{p, t} y_{t-1}\right) \\
C_{n} & =\sum_{t=1}^{n} \varepsilon_{p, t}^{2}-\left(\sum_{t=1}^{n} \varepsilon_{p, t} x_{p, t}^{\prime}\right)\left(\sum_{t=1}^{n} x_{p, t} x_{p, t}^{\prime}\right)^{-1}\left(\sum_{t=1}^{n} x_{p, t} \varepsilon_{p, t}\right)
\end{aligned}
$$

Now we have

$$
\begin{aligned}
\hat{\alpha}_{n}-1 & =A_{n} B_{n}^{-1} \\
\hat{\sigma}_{n}^{2} & =n^{-1}\left(C_{n}-A_{n}^{2} B_{n}^{-1}\right) \\
s\left(\hat{\alpha}_{n}\right)^{2} & =\hat{\sigma}_{n}^{2} B_{n}^{-1}
\end{aligned}
$$

where $\hat{\alpha}_{n}$ is the OLS estimator of $\alpha, \hat{\sigma}_{n}^{2}$ is the usual error variance estimator, and $s\left(\hat{\alpha}_{n}\right)$ is the estimated standard error for $\hat{\alpha}_{n}$. We also let

$$
\hat{\alpha}_{n}(1)=1-\sum_{k=1}^{p} \hat{\alpha}_{p, k}
$$

where $\hat{\alpha}_{p, k}$ 's are the OLS estimators of $\alpha_{k}$ 's in regression (3).

The statistics that we will consider in the paper are given by

$$
\begin{aligned}
S_{n} & =\frac{n\left(\hat{\alpha}_{n}-1\right)}{\hat{\alpha}_{n}(1)} \\
T_{n} & =\frac{\hat{\alpha}_{n}-1}{s\left(\hat{\alpha}_{n}\right)}
\end{aligned}
$$


Note that $S_{n}$ is a normalized unit root regression coefficient, and $T_{n}$ is the $t$-statistic for the unit root hypothesis. The tests based on $S_{n}$ and $T_{n}$ will be referred to respectively as the coefficient test and the $t$-test. They are extensions of the tests considered by Dickey and Fuller $(1979,1981)$ for the AR(1) model.

It is shown in Chang and Park (2001) that

Theorem 2.4 (Limiting Distributions of ADF Tests) Under Assumptions A1, $\mathrm{A} 2$ and $\mathrm{A} 3$, we have

$$
S_{n} \rightarrow \frac{\int_{0}^{1} W_{t} d W_{t}}{\int_{0}^{1} W_{t}^{2} d t}, \quad T_{n} \rightarrow_{d} \frac{\int_{0}^{1} W_{t} d W_{t}}{\left(\int_{0}^{1} W_{t}^{2} d t\right)^{1 / 2}}
$$

as $n \rightarrow \infty$, where $W$ is the standard Brownian motion.

The asymptotic null distributions of $S_{n}$ and $T_{n}$ are thus identical to those of the corresponding statistics studied in Dickey and Fuller $(1979,1981)$. They are tabulated in Fuller (1996).

The models with deterministic trends can be analyzed similarly. If the time series $\left(z_{t}\right)$ is given by

$$
z_{t}=\mu+y_{t} \quad \text { or } \quad z_{t}=\mu+\tau t+y_{t}
$$

and $\left(y_{t}\right)$ is generated as in (1), the unit root hypothesis can be tested in regression (3) run by the fitted values of $\left(y_{t}\right)$ obtained from the preliminary regression (10). Their distributions are given similarly as those in Theorem 2.4, respectively with demeaned and detrended Brownian motions

$$
W_{t}^{\mu}=W_{t}-\int_{0}^{1} W_{s} d s, \quad W_{t}^{\tau}=W_{t}+(6 t-4) \int_{0}^{1} W_{s} d s-(12 t-6) \int_{0}^{1} s W_{s} d s
$$

in place of standard Brownian motion $W$. Though we do not report the details, our results here can be easily extended to obtain the asymptotic theory for the unit root tests in models with deterministic trends.

\section{Asymptotics for the Bootstrap ADF Tests}

We now consider the sieve bootstraps for the ADF tests. In this section, we establish the bootstrap consistency of the tests and show that they are asymptotically valid. The results presented here are mainly theoretical. Various problems arising in practical implementation of the sieve bootstrap methodology are fully addressed in the next section. To concentrate on the theoretical aspects of the bootstrap ADF tests, the discussions on any empirical issues will be kept minimal in this section. Throughout the paper, the notation $*$ is used as usual to signify the bootstrap samples. Moreover, we use $\mathbf{P}^{*}$ and $\mathbf{E}^{*}$ respectively to denote the probability and expection conditional on the realization of original sample. 
To obtain bootstrap samples, we first let $u_{t}=\Delta y_{t}$ and fit the approximated autoregression

$$
u_{t}=\alpha_{1} u_{t-1}+\cdots+\alpha_{p} u_{t-p}+\varepsilon_{p, t}
$$

by the usual OLS regression. We denote by $\tilde{\alpha}_{p, k}$ 's the OLS estimators of $\alpha_{k}$ 's, and by $\left(\tilde{\varepsilon}_{p, t}\right)$ the OLS residuals in regression (11). It is important to base the bootstrap sampling on regression (11) with the restriction $\alpha=1$. As shown in Basawa et al. (1991a), the samples generated by regression (3) without the unit root restriction do not behave like unit root processes, and make the subsequent bootstrap procedures inconsistent. We may prefer, especially in small samples, to use the Yule-Walker method to estimate (11) since it always yields an autoregression that is invertible. Our subsequent results are applicable also for the Yule-Walker method, since it is asymptotically equivalent to the OLS method.

Now we construct the bootstrap sample for $\left(\varepsilon_{t}^{*}\right),\left(u_{t}^{*}\right)$ and $\left(y_{t}^{*}\right)$ as follows. First, resample $\left(\varepsilon_{t}^{*}\right)$ from the centered fitted residuals from the approximated AR (11), i.e., obtain iid samples $\left(\varepsilon_{t}^{*}\right)$ from the empirical distribution of

$$
\left(\tilde{\varepsilon}_{p, t}-\bar{\varepsilon}_{p, n}\right)_{t=1}^{n}
$$

where $\bar{\varepsilon}_{p, n}=(1 / n) \sum_{t=1}^{n} \tilde{\varepsilon}_{p, t}$. Next, generate $\left(u_{t}^{*}\right)$ from $\left(\varepsilon_{t}^{*}\right)$ using the fitted autoregression given by

$$
u_{t}^{*}=\tilde{\alpha}_{p, 1} u_{t-1}^{*}+\cdots+\tilde{\alpha}_{p, p} u_{t-p}^{*}+\varepsilon_{t}^{*}
$$

with appropriately chosen $p$-initial values of $\left(u_{t}^{*}\right)$. Finally, obtain $\left(y_{t}^{*}\right)$ by taking partial sumes of $\left(u_{t}^{*}\right)$, i.e.,

$$
y_{t}^{*}=y_{0}^{*}+\sum_{k=1}^{t} u_{k}^{*}
$$

with some initial value $y_{0}^{*}$. The choice of the initial value $y_{0}^{*}$ for $\left(y_{t}^{*}\right)$ does not affect the asymptotics as long as it is stochastically bounded, and we thus simply set it equal to zero for the subsequent development of our theory in this section. See discussions in the next section on the initializations of $\left(u_{t}^{*}\right)$ and $\left(y_{t}^{*}\right)$ in (12) and (13).

For the bootstrap ADF tests, we consider

$$
y_{t}^{*}=\alpha y_{t-1}^{*}+\sum_{k=1}^{p} \alpha_{k} \triangle y_{t-k}^{*}+\varepsilon_{t}^{*}
$$

and test for the unit root hypothesis $\alpha=1$. Similarly as before, we denote by $\hat{\alpha}_{n}^{*}$ and $s\left(\hat{\alpha}_{n}^{*}\right)$ respectively the OLS estimator for $\alpha$ and the standard error for $\hat{\alpha}_{n}^{*}$ obtained using the bootstrap samples. To define them more explicitly, we let

$$
x_{p, t}^{*}=\left(\triangle y_{t-1}^{*}, \ldots, \triangle y_{t-p}^{*}\right)^{\prime}
$$

and subsequently define

$$
\begin{aligned}
& A_{n}^{*}=\sum_{t=1}^{n} y_{t-1}^{*} \varepsilon_{t}^{*}-\left(\sum_{t=1}^{n} y_{t-1}^{*} x_{p, t}^{* \prime}\right)\left(\sum_{t=1}^{n} x_{p, t}^{*} x_{p, t}^{* \prime}\right)^{-1}\left(\sum_{t=1}^{n} x_{p, t}^{*} \varepsilon_{t}^{*}\right) \\
& B_{n}^{*}=\sum_{t=1}^{n} y_{t-1}^{* 2}-\left(\sum_{t=1}^{n} y_{t-1}^{*} x_{p, t}^{* \prime}\right)\left(\sum_{t=1}^{n} x_{p, t}^{*} x_{p, t}^{* \prime}\right)^{-1}\left(\sum_{t=1}^{n} x_{p, t}^{*} y_{t-1}^{*}\right)
\end{aligned}
$$


and the variance of the bootstrap sample $\left(\varepsilon_{t}^{*}\right)$, which is given by

$$
\tilde{\sigma}_{n}^{2}=\frac{1}{n} \sum_{t=1}^{n}\left(\tilde{\varepsilon}_{p, t}-\bar{\varepsilon}_{p, n}\right)^{2}
$$

Then we may write

$$
\begin{aligned}
& \hat{\alpha}_{n}^{*}-1=A_{n}^{*} B_{n}^{*-1} \\
& s\left(\hat{\alpha}_{n}^{*}\right)^{2}=\tilde{\sigma}_{n}^{2} B_{n}^{*-1}
\end{aligned}
$$

We also define

$$
\tilde{\alpha}_{n}(1)=1-\sum_{k=1}^{p} \tilde{\alpha}_{p, k}
$$

accordingly as $\hat{\alpha}_{n}(1)$ introduced in (7) before.

Now we consider the statistics

$$
\begin{aligned}
S_{n}^{*} & =\frac{n\left(\hat{\alpha}_{n}^{*}-1\right)}{\tilde{\alpha}_{n}(1)} \\
T_{n}^{*} & =\frac{\hat{\alpha}_{n}^{*}-1}{s\left(\hat{\alpha}_{n}^{*}\right)}
\end{aligned}
$$

corresponding to $S_{n}$ and $T_{n}$ introduced in (8) and (9) of the previous section. It is also possible to use $\hat{\alpha}_{n}^{*}(1)$ and $\hat{\sigma}_{n}^{2 *}$, the bootstrap counterparts to $\hat{\alpha}_{n}(1)$ and $\hat{\sigma}_{n}^{2}$, in place of $\tilde{\alpha}_{n}(1)$ and $\tilde{\sigma}_{n}^{2}$ for the construction of the bootstrap test statistics $S_{n}^{*}$ and $T_{n}^{*}$. They can be obtained from regression (14) in the same way that their counterparts are computed from regression (3). We may indeed show without difficulty that such replacements do not affect the limiting distributions of the statistics. For the theoretical analysis in the paper, however, we only consider $S_{n}^{*}$ and $T_{n}^{*}$ defined in (17) and (18). This is mainly for the expositional brevity. We have indeed investigated both versions of the statistics for the simulations reported in the next section.

To implement the bootstrap ADF tests, we repeat the bootstrap sampling for the given original sample and obtain $a_{n}(\lambda)$ and $b_{n}(\lambda)$ such that

$$
\mathbf{P}^{*}\left\{S_{n}^{*} \leq a_{n}(\lambda)\right\}=\mathbf{P}^{*}\left\{T_{n}^{*} \leq b_{n}(\lambda)\right\}=\lambda
$$

for any prescribed size level $\lambda$. The bootstrap ADF tests reject the null hypothesis of a unit root if

$$
S_{n} \leq a_{n}(\lambda), \quad T_{n} \leq b_{n}(\lambda)
$$

It will now be shown under appropriate conditions that the tests are asymptotically valid, i.e., they have asymptotic size $\lambda$. We do not analyze in the paper the randomness associated with the bootstrap sampling in computing the bootstrap critical values $a_{n}(\lambda)$ and $b_{n}(\lambda)$. We simply assume that enough number of bootstrap iterations is done to make it disappear or at least negligible. See Andrews and Buchinsky (1999) for a study on the number of bootstrap iterations to achieve the desired level of bootstrap sampling accuracy.

For the subsequent results in this section, we assume 
Assumption B1 Let $\left(\varepsilon_{t}\right)$ be a sequence of iid random variables such that $\mathbf{E} \varepsilon_{t}=0$, $\mathbf{E} \varepsilon_{t}^{2}=\sigma^{2}$ and $\mathbf{E}\left|\varepsilon_{t}\right|^{r}<\infty$ for some $r \geq 4$.

Assumption B2 Let $\pi(z) \neq 0$ for all $|z| \leq 1$, and $\sum_{k=0}^{\infty}|k|^{s}\left|\pi_{k}\right|<\infty$ for some $s \geq 1$.

Assumption B3a Let $p_{n} \rightarrow \infty$ and $p_{n}=o\left(n^{\kappa}\right)$ with $\kappa<1 / 2$ as $n \rightarrow \infty$.

Assumption B3b Let $p_{n}=c n^{\kappa}$ for some constant $c$ and $1 / r s<\kappa<1 / 2$.

Assumptions B1, B2 and B3a together will be refered to as Assumption (W), with 'W' standing for weak, and the set of Assumptions B1, B2 and B3b will be called as Assumption (S), with 'S' for strong.

The iid assumption in Assumption B1, instead of the martingale difference condition in Assumption A1, is made to make the usual bootstrap procedure meaningful. Assumption B2 is identical to Assumption A2. In the place of Assumption A3 for the expansion rate of $\mathrm{AR}$ order $p$, we impose either Assumption B3a or B3b. Both Assumptions B3a and B3b are stronger than Assumption A3. We will impose the condition in Assumption B3a to prove the consistency of the bootstrap ADF tests in the weak form, i.e., the convergence of conditional bootstrap distributions in probability. To establish the strong consistency or the a.s. convergence of conditional bootstrap distributions, we need a stronger condition in Assumption B3b. Notice that we only require $0<\kappa<1 / 2$, for the Gaussian model with $r=\infty$ or the finite order ARMA model with $s=\infty$. The condition is therefore not very stringent.

Remark 3.1: Bootstrap Stochastic Order Symbols It is convenient to introduce the symbols $o_{p}^{*}$ and $O_{p}^{*}$ for our development of bootstrap sample asymptotics, which correspond to $o_{p}$ and $O_{p}$ for the original sample asymptotics. Here we just define $o_{p}^{*}(1)$ and $O_{p}^{*}(1)$. The extension of our definition to $o_{p}^{*}\left(c_{n}\right)$ and $O_{p}^{*}\left(c_{n}\right)$ for some nonconstant numerical sequence $\left(c_{n}\right)$, however, should be obvious. Let $\left(X_{n}^{*}\right)$ be a sequence of bootstrapped statistics. We define $X_{n}^{*}=o_{p}^{*}(1)$ a.s. and in $\mathbf{P}$ to imply respectively that

$$
\mathbf{P}^{*}\left\{\left|X_{n}^{*}\right|>\epsilon\right\} \rightarrow 0 \text { a.s. and in } \mathbf{P}
$$

for any $\epsilon>0$. Moreover, if for every $\epsilon>0$ given there exists a constant $M>0$ such that for all large $n$

$$
\mathbf{P}^{*}\left\{\left|X_{n}^{*}\right|>M\right\}<\epsilon
$$

we write $X_{n}^{*}=O_{p}^{*}(1)$ a.s. or in $\mathbf{P}$ depending upon whether the condition holds a.s. (with probability one), or holds in $\mathbf{P}$ (with probability arbitrarily close to one).

As might well be expected, the bootstrap stochastic orders $o_{p}^{*}$ and $O_{p}^{*}$ of the bootstrapped statistics $\left(X_{n}^{*}\right)$ can be obtained directly from the sample stochastic order of $\mathbf{E}^{*}\left|X_{n}^{*}\right|$ (or $\mathbf{E}^{*}\left|X_{n}^{*}\right|^{r}$ for some $r>0$ ) using Markov inequality. It is indeed easy to see that, if $\mathbf{E}^{*}\left|X_{n}^{*}\right| \rightarrow 0$ a.s. (or in $\mathbf{P}$ ), then $X_{n}^{*}=o_{p}^{*}(1)$ a.s. (or in $\mathbf{P}$ ). One may likewise readily show that, if $\mathbf{E}^{*}\left|X_{n}^{*}\right|=O(1)$ a.s. (or in $\mathbf{P}$, i.e., $O_{p}(1)$ ), then $X_{n}^{*}=O_{p}^{*}(1)$ a.s. (or in $\left.\mathbf{P}\right)$. 
Many of the well known results for $o_{p}$ and $O_{p}$ naturally extend to $o_{p}^{*}$ and $O_{p}^{*}$. Here we list some of them as a lemma.

Lemma 3.1 Let $o_{p}^{*}$ and $O_{p}^{*}$ be defined as in Remark 3.1 above. Then we have

(a) $O_{p}^{*}(1) o_{p}^{*}(1)=o_{p}^{*}(1)$

(b) $O_{p}^{*}(1) O_{p}^{*}(1)=O_{p}^{*}(1)$

(c) $O(1) o_{p}^{*}(1)=o(1) O_{p}^{*}(1)=o_{p}^{*}(1)$

for orders $o_{p}^{*}, O_{p}^{*}, o$ and $O$ defined in both a.s. and in $\mathbf{P}$ senses.

The following lemmas are needed for the derivation of the limit distributions for the bootstrap ADF tests. Let

$$
\tilde{\pi}_{n}(1)=\tilde{\alpha}_{n}(1)^{-1}
$$

where $\tilde{\alpha}_{n}(1)$ is defined in (16).

Lemma 3.2 Under Assumption (W), we have
(a) $\frac{1}{n} \sum_{t=1}^{n} y_{t-1}^{*} \varepsilon_{t}^{*}=\tilde{\pi}_{n}(1) \frac{1}{n} \sum_{t=1}^{n} w_{t-1}^{*} \varepsilon_{t}^{*}+o_{p}^{*}(1)$ a.s.
(b) $\frac{1}{n^{2}} \sum_{t=1}^{n} y_{t-1}^{* 2}=\tilde{\pi}_{n}(1)^{2} \frac{1}{n^{2}} \sum_{t=1}^{n} w_{t-1}^{* 2}+o_{p}^{*}(1)$ a.s.

Lemma 3.3 We have

(a) $\left\|\left(\frac{1}{n} \sum_{t=1}^{n} x_{p, t}^{*} x_{p, t}^{* \prime}\right)^{-1}\right\|=O_{p}^{*}(1)$ a.s. (or in $\left.\mathbf{P}\right)$ under Assumption (S) (or $(\mathrm{W})$ )
(b) $\left\|\sum_{t=1}^{n} x_{p, t}^{*} y_{t-1}^{*}\right\|=O_{p}^{*}\left(n p^{1 / 2}\right)$ a.s. under Assumption (W)
(c) $\left\|\sum_{t=1}^{n} x_{p, t}^{*} \varepsilon_{t}^{*}\right\|=O_{p}^{*}\left(n^{1 / 2} p^{1 / 2}\right)$ a.s. under Assumption (W)

Now we have from Lemmas 3.1 and 3.3

$$
\begin{aligned}
& \left|\left(\sum_{t=1}^{n} y_{t-1}^{*} x_{p, t}^{* \prime}\right)\left(\sum_{t=1}^{n} x_{p, t}^{*} x_{p, t}^{* \prime}\right)^{-1}\left(\sum_{t=1}^{n} x_{p, t}^{*} \varepsilon_{t}^{*}\right)\right| \\
& \leq\left\|\sum_{t=1}^{n} y_{t-1}^{*} x_{p, t}^{* \prime}\right\|\left\|\left(\sum_{t=1}^{n} x_{p, t}^{*} x_{p, t}^{* \prime}\right)^{-1}\right\|\left\|\sum_{t=1}^{n} x_{p, t}^{*} \varepsilon_{t}^{*}\right\|=O_{p}^{*}\left(n^{1 / 2} p\right)
\end{aligned}
$$

which, together with Lemma 3.2, implies that

$$
\begin{aligned}
& n^{-1} A_{n}^{*}=\tilde{\pi}_{n}(1) \frac{1}{n} \sum_{t=1}^{n} w_{t-1}^{*} \varepsilon_{t}^{*}+o_{p}^{*}(1) \text { a.s. }(\text { or in } \mathbf{P}) \\
& \left.n^{-2} B_{n}^{*}=\tilde{\pi}_{n}(1)^{2} \frac{1}{n^{2}} \sum_{t=1}^{n} w_{t-1}^{* 2}+o_{p}^{*}(1) \text { a.s. (or in } \mathbf{P}\right)
\end{aligned}
$$


under Assumption (S) (or (W)).

Remark 3.2: Notation for Bootstrap Asymptotics For a sequence of bootstrapped statistics $\left(X_{n}^{*}\right)$, we write

$$
X_{n}^{*} \rightarrow d^{*} X \text { a.s. (or in } \mathbf{P} \text { ) }
$$

if the conditional distribution of $\left(X_{n}^{*}\right)$ weakly converges to that of $X$ a.s. (or in $\mathbf{P}$ ). Here it is assumed that the limiting random variable $X$ has distribution independent of the original sample realization. We may therefore simply write $\mathbf{P}$ and $\mathbf{E}$ for the probability and expection associated with $X$. Let $P_{X}=\mathbf{P} X^{-1}$ be the distribution of $X$. Then it follows that $X_{n}^{*} \rightarrow d^{*} X$ a.s. if and only if $\mathbf{E}^{*} f\left(X_{n}^{*}\right) \rightarrow$ a.s. $\mathbf{E} f(X)$ for every $f$ bounded and uniformly continuous $P_{X}$ a.s. [see, e.g., Parthasarathy (1967, Theorem 6.6)]. Similarly, we have $X_{n}^{*} \rightarrow d^{*} X$ in $\mathbf{P}$ if and only if $\mathbf{E}^{*} f\left(X_{n}^{*}\right) \rightarrow{ }_{p} \mathbf{E} f(X)$ for every $f$ bounded and uniformly continuous $P_{X}$ a.s. For a sequence of bootstrapped statistics $\left(X_{n}^{*}\right)$ which weakly converges a.s. (or in $\mathbf{P}$ ), we may easily show that $X_{n}^{*}=O_{p}^{*}(1)$ a.s. (or in $\mathbf{P}$ ). Moreover, if $X_{n}^{*} \rightarrow d^{*} X$ a.s. (or in $\mathbf{P}$ ), then $X_{n}^{*}+Y_{n}^{*} \rightarrow d^{*} X$ a.s. (or in $\mathbf{P}$ ) for any $\left(Y_{n}^{*}\right)$ such that $Y_{n}^{*}=o_{p}^{*}(1)$ a.s. (or in $\mathbf{P}$ ).

Under Assumption (W), the parameter estimates $\tilde{\sigma}_{n}^{2}$ and $\tilde{\pi}_{n}(1)$ defined in (15) and (20) are strongly consistent.

Lemma 3.4 Under Assumption (W), we have $\tilde{\sigma}_{n}^{2} \rightarrow$ a.s. $\sigma^{2}$ and $\tilde{\pi}_{n}(1) \rightarrow a . s . \pi(1)$ as $n \rightarrow \infty$.

Moreover, as shown in Park (1999), we have $n^{-1} \sum_{t=1}^{n} w_{t-1}^{*} \varepsilon_{t}^{*}, n^{-2} \sum_{t=1}^{n} w_{t-1}^{* 2}=O_{p}^{*}(1)$ a.s. under Assumption (W). Therefore, it can be deduced that

$$
S_{n}^{*}=\frac{\frac{1}{n} \sum_{t=1}^{n} w_{t-1}^{*} \varepsilon_{t}^{*}}{\frac{1}{n^{2}} \sum_{t=1}^{n} w_{t-1}^{* 2}}+o_{p}^{*}(1), T_{n}^{*}=\frac{\frac{1}{n} \sum_{t=1}^{n} w_{t-1}^{*} \varepsilon_{t}^{*}}{\sigma\left(\frac{1}{n^{2}} \sum_{t=1}^{n} w_{t-1}^{* 2}\right)^{1 / 2}}+o_{p}^{*}(1) \text { a.s. }(\text { or in } \mathbf{P})
$$

under Assumption (S) (or $(\mathrm{W})$ ). The limiting distributions of the bootstrap ADF tests may now be easily obtained using the result in Park (1999).

Theorem 3.5 (Limiting Distributions of Bootstrap ADF Tests) We have respectively under Assumptions (S) and (W)

$$
S_{n}^{*} \rightarrow d^{*} \frac{\int_{0}^{1} W_{t} d W_{t}}{\int_{0}^{1} W_{t}^{2} d t}, T_{n}^{*} \rightarrow d^{*} \frac{\int_{0}^{1} W_{t} d W_{t}}{\left(\int_{0}^{1} W_{t}^{2} d t\right)^{1 / 2}} \text { a.s. and in } \mathbf{P}
$$

as $n \rightarrow \infty$, where $W$ is the standard Brownian motion. 
Theorem 3.4 shows that the bootstrap statistics $S_{n}^{*}$ and $T_{n}^{*}$ have the same limiting distributions as the corresponding sample statistics $S_{n}$ and $T_{n}$. It implies, in particular, that the bootstrap ADF tests are asymptotically valid. This will be explained below.

Denote by $S$ and $T$ the weak limits of $S_{n}$ and $T_{n}$ respectively, and define $a(\lambda)$ and $b(\lambda)$ to be the asymptotic critical values of the size $\lambda$ tests based on $S_{n}$ and $T_{n}$, i.e.,

$$
\mathbf{P}\{S \leq a(\lambda)\}=\mathbf{P}\{T \leq b(\lambda)\}=\lambda
$$

Since the distribution of $S$ and $T$ are absolutely continuous with respect to Lebesque measure, we have from Theorem 3.4

$$
\left.\mathbf{P}^{*}\left\{S_{n}^{*} \leq a(\lambda)\right\}, \mathbf{P}^{*}\left\{T_{n}^{*} \leq b(\lambda)\right\} \rightarrow \lambda \text { a.s. (or in } \mathbf{P}\right)
$$

under Assumption (S) (or (W)), and the results in (21) imply

$$
\left(a_{n}(\lambda), b_{n}(\lambda)\right) \rightarrow(a(\lambda), b(\lambda)) \text { a.s. }(\text { or in } \mathbf{P})
$$

where $a_{n}(\lambda)$ and $b_{n}(\lambda)$ are the size $\lambda$ bootstrap critical values defined in (19). Consequently, we have under Assumption (W)

$$
\mathbf{P}\left\{S_{n} \leq a_{n}(\lambda)\right\}, \mathbf{P}\left\{T_{n} \leq b_{n}(\lambda)\right\} \rightarrow \lambda
$$

which proves that the bootstrap ADF tests have size $\lambda$ asymptotically.

Our bootstrap theory here easily extends to the tests for a unit root in models with deterministic trends, such as those introduced in (10). It is indeed quite

straightforward to establish the bootstrap consistency for the ADF tests applied to the demeaned and detrended time series, using the results obtained in this section. The bootstrap ADF tests are therefore valid and applicable also for the models with deterministic trends.

\section{Practical Issues and Simulation Results}

There are several practical issues on the actual bootstrap procedures for the tests proposed in the paper. They are listed and addressed below.

(a) We may rely on various selection criteria such as AIC and BIC to fix the orders of the approximated autoregressions (3) and (11). If it is known that the true model is generated by a finite order autoregression, the order selection based on BIC is consistent, and therefore, it might be preferred. Such a case, however, is rare in practical applications. True model is unknown, and not likely to be given exactly by a finite order autoregression. We may thus use AIC, in favor of BIC, since it leads to an symptotically efficient choice of the optimal order for a class of infinite order autoregressive processes [see Brockwell and Davis (1991)]. The order selection for regression (3) has previously been addressed by many authors including Stock (1994) and Ng and Perron (2000). 
(b) We may use other methods to estimate the approximated autoregression (11). In particular, the Yule-Walker method may be preferred to the OLS method in small samples, since it always yields an invertible autoregression [see, e.g., Brockwell and Davis (1991, Sections 8.1 and 8.2)]. As the sample size increases, however, the problem of noninvertibility for the latter vanishes a.s., and the two methods become equivalent.

(c) It is necessary to initialize the autoregression in (12) to obtain bootstrap samples for $\left(u_{t}^{*}\right)$. We may use the first $p$-values of $\left(u_{t}\right)$ and generate the samples $\left(u_{t}^{*}\right)$ conditional on them. Strictly speacking, however, this would not produce stationary autoregressive processes. To get bootstrap samples that are stationary, we may generate large number of values for $\left(u_{t}^{*}\right)$ and throw the first generated values away. If the bootstrap samples are generated as such, the initialization becomes unimportant and we may start from zeros.

(d) To generate the bootstrap samples $\left(y_{t}^{*}\right)$, we also need initialization in (13). An obvious choice would be to use the initial value $y_{0}$ of $\left(y_{t}\right)$, and generate the bootstrap samples conditional on $y_{0}$. The effect of the initial value becomes negligible asymptotically, as mentioned earler in the previous section. However, it may affect finite sample performance of the bootstrap. If the mean or linear time trend is maintained as in (10) and the unit root test is performed using the demeaned or detrended data, then the effect of the initial value $y_{0}^{*}$ of the bootstrap sample would disappear. We may therefore just set $y_{0}^{*}=0$.

(e) The bootstrap statistics $S_{n}^{*}$ and $T_{n}^{*}$ defined in (17) and (18) involve $\tilde{\alpha}_{n}(1)$ and $\tilde{\sigma}_{n}^{2}$. These are the population parameters for the bootstrap samples $\left(\varepsilon_{t}^{*}\right)$ and $\left(u_{t}^{*}\right)$, corresponding to $\alpha(1)$ and $\sigma^{2}$ for the original samples $\left(\varepsilon_{t}\right)$ and $\left(u_{t}\right)$. Obviously, we may use the bootstrap estimates $\hat{\alpha}_{n}^{*}$ and $\hat{\sigma}_{n}^{2 *}$, say, for each bootstrap iteration to construct the statistics $S_{n}^{*}$ and $T_{n}^{*}$. It is indeed straightforward to show that two versions of the bootstrap tests are asymptotically equivalent. However, they may behave differently in finite samples.

We conduct a set of simulations to investigate these and other related issues, along with the general finite sample performances of the bootstrap ADF tests considered in the paper. The issues listed above will be given a particular attention in the subsequent discussions on our simulation results.

For the simulation, we consider $\left(y_{t}\right)$ given by the model $(1)$ with $\left(u_{t}\right)$ generated by

$$
u_{t}=\varepsilon_{t}+\beta \varepsilon_{t-1}
$$

where $\left(\varepsilon_{t}\right)$ are iid $\mathbf{N}\left(0, \sigma^{2}\right)$. The test statistics are invariant with respect to the value of $\sigma^{2}$, and therefore, we set $\sigma^{2}=1$ in our simulation. The parameter values for the moving average coefficient $\beta$ in $(22)$ are chosen to be $\beta=-0.8,-0.4,0,0.4,0.8$. To examine the finite sample size, we set $\alpha=1$. For the finite sample power comparisons, we look at the values $\alpha=0.95$ and 0.90 . Three different size tests, $1 \%, 5 \%$ and $10 \%$, are examined, and the samples of sizes $n=50$ and 100 are considered. The reported results are based on 5,000 simulation iterations with the bootstrap critical values computed using 5,000 bootstrap repetitions. 
We use the AIC criterion to select the orders of the approximated autoregressions (3) and (11). ${ }^{3}$ As explained earlier, AIC may be considered to be more appropriate than BIC when, as is in our case, the true model is not generated by any finite order autoregression. The maximum lag length is set to be $10 \log _{10}(n)$, the default value for SPLUS, which yields 16.99, and 20.00 respectively for the samples of size 50 and 100. Using BIC instead of AIC generally gives higher rejection probabilities under both the null and alternative hypotheses. A reversed tendancy has been observed when we increase the number of maximum lag length. The use of AIC with no restriction on the maximum lag length yields the lowest rejection probabilities. The highest rejection probabilities are observed with the application of BIC with smallest maximum lag length. However, the choice of the selection criteria and the maximum lag length do not seem to affect the discriminatory powers of the tests. Their effects are rather uniform regardless of the presence or absence of the unit root.

We also evaluate the procedure by $\mathrm{Ng}$ and Perron (2000) to select the order of autoregression (3). Their procedure lowers the rejection rates drastically for models with large negative moving average coefficients. It, however, leads to somewhat severe under-rejection of the unit root hypothesis when the moving average coefficient becomes large positive. The application of bootstrap seems to improve the finite sample performance of the tests also in this context, but such tendency still persists. The resulting bootstrap tests make the rejection rates even lower substantially for moving average innovations with large negative coefficients, but the under-rejection for those with large positive coefficients become slightly worse.

For the reported simulation results, we use the OLS method to estimate the approximated autoregression (11). Our unreported simulations, however, show that the results based on the Yule-Walker estimation of regression (11) are not much different even for samples of size 50 or smaller. To initialize the bootstrap sample $\left(u_{t}^{*}\right)$, we use the $p$-initial values of $\left(u_{t}\right)$. We have also tried to generate $\left(u_{t}^{*}\right)$ twice the size of the required samples and discard the first half of the observations. This was to obtain samples which look more likely to be drawn from a stationary process. The simulation results, however, were not significantly different. For the initialization of $\left(y_{t}^{*}\right)$, we simply set $y_{0}^{*}=0$. Finally, we considered two versions of the bootstrap ADF tests: one with $\tilde{\alpha}_{n}(1)$ and $\tilde{\sigma}_{n}^{2}$, and the other with $\hat{\alpha}_{n}^{*}(1)$ and $\hat{\sigma}_{n}^{2 *}$. Their relative performances are somewhat dependent upon the values of the moving average coefficient $\beta$. However, we find that the latter with the bootstrapped parameter estimates generally perform slightly better.

Our simulation results are summarized in Tables 1 and 2. The reported results are for the tests with demeaned $\left(y_{t}\right) .{ }^{4}$ Tables 1 and 2 present, respectively, the rejection probabilities for the $1 \%, 5 \%$ and $10 \%$ tests. The bootstrap ADF tests $S_{n}^{*}$ and $T_{n}^{*}$ are compared with the corresponding usual ADF tests $S_{n}$ and $T_{n}$. In general, the bootstrap procedure seems to improve the finite sample sizes of the ADF tests, at no

\footnotetext{
${ }^{3}$ The orders of regressions (3) and (11) are therefore different in general, though both are denoted by $p$ and assumed for expositional simplicity to be identical in the text.

${ }^{4}$ The GLS demeaning suggested by Elliott, Rothenberg and Stock (1996) is also considered though the detailed results are not reported. In general, it increases the powers of the tests, but does neither improve nor disimprove the finite sample size corrections of bootstraps.
} 
cost in terms of powers. The empirical sizes of tests based on $S_{n}^{*}$ and $T_{n}^{*}$ are relatively much closer to the nominal sizes than the usual tests relying on $S_{n}$ and $T_{n}$, even for samples as small as $n=50$. In particular, the size corrections by bootstrap appear to be drastic, when the moving average coefficient takes large negative values. It is well known that the ADF tests have large size distortions in such cases.

The size performance of the coefficient test $S_{n}$ improves more noticeably when we use the bootstrapped critical values and consider $S_{n}^{*}$. The bootstrap improvement for the $t$-test, i.e., $T_{n}^{*}$ over $T_{n}$ is relatively smaller in most cases. It appears that the coefficient test has more room for improvement using bootstrap methodology. On the other hand, once corrected for sizes using bootstraps, the coefficient test $S_{n}^{*}$ offers more power than the $t$-test $T_{n}^{*}$, except for the case that we have large negative moving average coefficients. All our results reported here apply to the detrended case, as well as the demeaned case, at least qualitatively. The results for the tests with detrended $\left(y_{t}\right)$ are therefore not reported in detail.

\section{Conclusion}

We consider in this paper the bootstrap procedures for the ADF tests for a unit root. We establish the bootstrap consistency of the tests, and show that the bootstrap ADF tests are asymptotically valid. The finite sample performances of the bootstrap ADF tests are investigated and compared with the usual ADF tests through simulations. The bootstrap tests are found to have finite sample sizes that are generally much closer to their nominal values, especially for models with large negative moving average coefficients. The bootstrap theory developed in the paper is also directly applicable for other types of unit root tests arising in many different contexts, including panel unit root models with cross-sectional dependencies and unit root tests using covariates. The extension of our theory to more general nonstationary model is also possible, and appears to be useful to analyze models such as cointegrating regressions and error correction models. Such extensions and applications are under way by the authors.

\section{Mathematical Proofs}

Proof of Lemma 3.1 The proofs are essentially identical to those for their sample analogues such as $O_{p}(1) o_{p}(1)=o_{p}(1)$ and $O_{p}(1) O_{p}(1)=O_{p}(1)$. The details are therefore omitted.

For the proofs for the bootstrap asymptotics given in Lemmas 3.2 and 3.3, we first prove some useful lemmas.

Lemma A1 Let Assumption (W) hold, and define

$$
\mathbf{E}^{*}\left|\varepsilon_{t}^{*}\right|^{2}=\sigma_{*}^{2} \quad \text { and } \quad \mathbf{E}^{*}\left|u_{t}^{*}\right|^{2}=\Gamma_{0}^{*}
$$

Then we have $\sigma_{*}^{2} \rightarrow$ a.s. $\sigma^{2}$ and $\Gamma_{0}^{*} \rightarrow$ a.s. $\Gamma_{0}$ as $n \rightarrow \infty$. 
Proof of Lemma A1 See Proof of Lemma 4.1 in Park (1999).

Lemma A2 Let Assumption (W) hold. If we denote respectively by $f$ and $f^{*}$ the spectral densities of $\left(u_{t}\right)$ and $\left(u_{t}^{*}\right)$, then we have

$$
\sup _{\lambda}\left|f^{*}(\lambda)-f(\lambda)\right|=o(1) \text { a.s. }
$$

for large $n$. Moreover, if we let $\left(\Gamma_{k}\right)$ and $\left(\Gamma_{k}^{*}\right)$ respectively be the autocovariance functions of $\left(u_{t}\right)$ and $\left(u_{t}^{*}\right)$, then

$$
\sum_{k=-\infty}^{\infty} \Gamma_{k}^{*}=\sum_{k=-\infty}^{\infty} \Gamma_{k}+o(1) \text { a.s. }
$$

for large $n$.

Proof of Lemma A2 Note that

$$
f^{*}(\lambda)=\frac{\sigma_{*}^{2}}{2 \pi}\left|1-\sum_{k=1}^{p} \tilde{\alpha}_{p, k} e^{i k \lambda}\right|^{-2}
$$

and as shown in Park (1999, Proofs of Lemmas 3.2 and 4.1)

$$
\sigma_{*}^{2}=\frac{1}{n} \sum_{t=1}^{n}\left(\tilde{\varepsilon}_{p, t}-\frac{1}{n} \sum_{t=1}^{n} \tilde{\varepsilon}_{p, t}\right)^{2}=\frac{1}{n} \sum_{t=1}^{n} \tilde{\varepsilon}_{p, t}^{2}+o_{p}(1)
$$

The stated result in the first part now follows from An, Chen and Hannan (1982, Theorem 5, p928). To deduce the result in the second part, we just note that

$$
\sum_{k=-\infty}^{\infty} \Gamma_{k}=2 \pi f(0) \text { and } \sum_{k=-\infty}^{\infty} \Gamma_{k}^{*}=2 \pi f^{*}(0)
$$

The proof is therefore complete.

Lemma A3 We have

$$
\mathbf{E}^{*}\left|\varepsilon_{t}^{*}\right|^{4}=O_{p}(1) \text { or } O(1) \text { a.s. }
$$

under Assumption (W) or (S) respectively.

Proof of Lemma A3 The stated results follow directly from Park (1999). We use his notation in the proof of Lemma 3.2. Applying his result with $r=4$, we have

$$
\mathbf{E}^{*}\left|\varepsilon_{t}^{*}\right|^{4} \leq c\left(A_{n}+B_{n}+C_{n}+D_{n}\right)
$$

where $c$ is some constant. As they have shown, $A_{n}=O(1)$ a.s., $\mathbf{E} B_{n}=o\left(p^{-r s}\right)$, $C_{n} \leq 2^{r-1}\left(C_{1 n}+C_{2 n}\right)$ with $C_{1 n}=o(1)$ a.s. and $\mathbf{E} C_{2 n}=o\left(p^{-r s}\right)$ and $D_{n}=o(1)$ a.s. Now note that $B_{n}=o(1)$ a.s. and $C_{2 n}=o(1)$ a.s. under our condition in Assumption $\mathrm{B} 3 \mathrm{~b}$, since $o\left(p^{-r s}\right)=o\left(n^{-1-\delta}\right)$ for some $\delta>0$. 
Lemma A4 Define

$$
M_{n}^{*}(i, j)=\mathbf{E}^{*}\left[\sum_{t=1}^{n}\left(u_{t-i}^{*} u_{t-j}^{*}-\Gamma_{i-j}^{*}\right)\right]^{2}
$$

Then we have

$$
M_{n}^{*}(i, j)=O_{p}(n) \text { or } O(n) \text { a.s. }
$$

uniformly in $i$ and $j$, under Assumption (W) or (S) respectively.

Proof of Lemma A4 From Berk (1974, Equations (2.10) and (2.11), p491), we have

$$
M_{n}^{*}(i, j) \leq n\left[2 \sum_{k=-\infty}^{\infty} \Gamma_{k}^{*}+\left|K_{4}^{*}\right|\left(\sum_{k=0}^{\infty} \tilde{\pi}_{p, k}^{2}\right)^{2}\right]
$$

for all $i$ and $j$, where $K_{4}^{*}$ is the fourth cumulant of $\left(\varepsilon_{t}^{*}\right)$. The stated results now follow from Lemmas A2 and A3.

We now prove the results for the bootstrap sample asymptotics stated in Lemmas 3.1, 3.2 and 3.3. Let $\tilde{\alpha}_{n}(1)$ and $\tilde{\pi}_{n}(1)$ be defined as in (16) and (20). Then we may easily deduce from regression (12) that

$$
\begin{aligned}
u_{t}^{*} & =\frac{1}{\tilde{\alpha}_{n}(1)} \varepsilon_{t}^{*}+\frac{1}{\tilde{\alpha}_{n}(1)} \sum_{k=1}^{p}\left(\sum_{i=k}^{p} \tilde{\alpha}_{p, i}\right)\left(u_{t-k}^{*}-u_{t-k+1}^{*}\right) \\
& =\tilde{\pi}_{n}(1) \varepsilon_{t}^{*}+\left(\bar{u}_{t-1}^{*}-\bar{u}_{t}^{*}\right)
\end{aligned}
$$

where $\bar{u}_{t}^{*}=\tilde{\pi}_{n}(1) \sum_{k=1}^{p}\left(\sum_{i=k}^{p} \tilde{\alpha}_{p, i}\right) u_{t-k+1}^{*}$ and therefore,

$$
y_{t}^{*}=\sum_{k=1}^{t} u_{k}^{*}=\tilde{\pi}_{n}(1) w_{t}^{*}+\left(\bar{u}_{0}^{*}-\bar{u}_{t}^{*}\right)
$$

where $w_{t}^{*}=\sum_{k=1}^{t} \varepsilon_{k}^{*}$.

Proof of Lemma 3.2 For Part (a), we write

$$
\sum_{t=1}^{n} y_{t-1}^{*} \varepsilon_{t}^{*}=\tilde{\pi}_{n}(1) \sum_{t=1}^{n} w_{t-1}^{*} \varepsilon_{t}^{*}+\tilde{u}_{0}^{*} \sum_{t=1}^{n} \varepsilon_{t}^{*}-\sum_{t=1}^{n} \tilde{u}_{t-1}^{*} \varepsilon_{t}^{*}
$$

and notice that

$$
\begin{gathered}
\mathbf{E}^{*}\left(\sum_{t=1}^{n} \varepsilon_{t}^{*}\right)^{2}=n \sigma_{*}^{2}=O(n) \text { a.s. } \\
\mathbf{E}^{*}\left(\sum_{t=1}^{n} \tilde{u}_{t-1}^{*} \varepsilon_{t}^{*}\right)^{2}=n \sigma_{*}^{2} \tilde{\Gamma}_{0}^{*}=O(n) \text { a.s. }
\end{gathered}
$$

where $\tilde{\Gamma}_{0}^{*}=\mathbf{E}^{*} \tilde{u}_{t}^{* 2}$ and other notation was defined earlier. The stated result now follows immediately. For Part (b), see Park (1999, Theorem 3.3). 
Proof of Lemma 3.3 We will show that

(a) $\mathbf{E}^{*}\left\|\left(\frac{1}{n} \sum_{t=1}^{n} x_{p, t}^{*} x_{p, t}^{* \prime}\right)^{-1}\right\|=O_{p}(1)$ or $O(1)$ a.s. under Assumptions (W) and (S), respectively.

(b) $\mathbf{E}^{*}\left\|\sum_{t=1}^{n} x_{p, t}^{*} y_{t-1}^{*}\right\|=O\left(n p^{1 / 2}\right)$ a.s. under Assumption $(\mathrm{W})$.

(c) $\mathbf{E}^{*}\left\|\sum_{t=1}^{n} x_{p, t}^{*} \varepsilon_{t}^{*}\right\|=O\left(n^{1 / 2} p^{1 / 2}\right)$ a.s. under Assumptions (W).

from which the stated results follow immediately.

We first prove Part (a). If we let $\underset{p p}{\stackrel{*}{*}}=\left(\Gamma_{i-j}^{*}\right)_{i, j=1}^{p}$, then it follows directly from Lemma A4 that

$$
\mathbf{E}^{*}\left\|\frac{1}{n} \sum_{t=1}^{n} x_{p, t}^{*} x_{p, t}^{* \prime}-{ }_{p p}^{*}\right\|^{2}=O_{p}\left(n^{-1} p^{2}\right) \text { or } O\left(n^{-1} p^{2}\right) \text { a.s. }
$$

under Assumption (W) or (S) respectively. Moreover, we have

$$
\|\stackrel{*-1}{p p}\| \leq\left[2 \pi\left(\inf _{\lambda} f^{*}(\lambda)\right)\right]^{-1}=O(1) \text { a.s. }
$$

under Assumption (W), due to Lemma A3. The stated result can now be easily obtained from the inequality

$$
\left|\mathbf{E}^{*}\left\|\left(\frac{1}{n} \sum_{t=1}^{n} x_{p, t}^{*} x_{p, t}^{* \prime}\right)^{-1}\right\|-\|\stackrel{*-1}{p p}\|\right| \leq \mathbf{E}^{*}\left\|\left(\frac{1}{n} \sum_{t=1}^{n} x_{p, t}^{*} x_{p, t}^{* \prime}\right)^{-1}-{ }_{p p}^{*-1}\right\|
$$

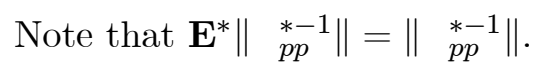

The proof for Part (b) is entirely analogous to that for Part (b) of Lemma 3.2 in Chang and Park (2001), given the results in Lemmas A2 and A4. Using the same notation (with * for the bootstrapped quantities) as in the proof of Part (b) of Lemma 3.2 in Chang and Park (2001), we may show

$$
R_{1 n}^{*}, R_{2 n}^{*}=O(n)+O_{p}^{*}\left(n^{1 / 2} p\right) \text { in } \mathbf{P} \text { or a.s. }
$$

under Assumption (W) or (S). Note that

$$
\sum_{k=-\infty}^{\infty} \Gamma_{k}^{*}=O(1) \text { a.s. }
$$

under Assumption (W).

For Part (c), note that we have for all $k, 1 \leq k \leq p$,

$$
\mathbf{E}^{*}\left(\sum_{t=1}^{n} u_{t-k}^{*} \varepsilon_{t}^{*}\right)^{2}=n \sigma_{*}^{2} \Gamma_{0}^{*}
$$

and therefore

$$
\mathbf{E}^{*}\left\|\sum_{t=1}^{n} x_{p, t}^{*} \varepsilon_{t}^{*}\right\|^{2}=n p \sigma_{*}^{2} \Gamma_{0}^{*}
$$

The stated result now follows immediately from Lemma A1. 
Proof of Lemma 3.4 The stated results follow from Lemma 3.1 in Park (1999).

Proof of Theorem 3.5 Given the results following Lemma 3.4, the limiting distributions of the bootstrapped statistics $S_{n}^{*}$ and $T_{n}^{*}$ can be obtained directly from Park (1999). Note that we have from Lemma 3.2 of Park (1999)

$$
\frac{1}{n} \sum_{t=1}^{n} w_{t-1}^{*} \varepsilon_{t}^{*} \rightarrow d^{*} \int_{0}^{1} W_{t} d W_{t} \text { a.s., } \frac{1}{n^{2}} \sum_{t=1}^{n} w_{t-1}^{* 2} \rightarrow d^{*} \int_{0}^{1} W_{t}^{2} d t \text { a.s. }
$$

under Assumption (W).

\section{References}

An, H.-Z., Z.-G. Chen and E.J. Hannan (1982). "Autocorrelation, autoregression and autoregressive approximation," Annals of Statistics 10, 926-936 (Corr: 11, p.1018).

Andrews, D.W.K. and M. Buchinsky (1999). "On the number of bootstrap repetitions for bootstrap standard errors, confidence intervals, and tests," Econometrica, forthcoming.

Basawa, I.V., A.K. Mallik, W.P. McCormick, J.H. Reeves and R.L. Taylor (1991a). "Bootstrapping unstable first-order autoregressive processes," Annals of Statistics 19, 1098-1101.

Basawa, I.V., A.K. Mallik, W.P. McCormick, J.H. Reeves and R.L. Taylor (1991b). "Bootstrap test of significance and sequential bootstrap estimation for unstable first order autoregressive processes," Communications in Statistics - Theory and Methods 20, 1015-1026.

Berk, K.N. (1974). "Consistent autoregressive spectral estimates," Annals of Statistics 2, 489-502.

Bickel, P.J. and P. Bühlmann (1999). "A new mixing notion and functional central limit theorems for a sieve bootstrap in time series," Bernoulli 5, 413-446.

Brockwell, P.J. and R.A. Davis (1991). Time Series: Theory and Methods, 2nd ed. Springer-Verlag: New York.

Bühlmann, P. (1997). "Sieve bootstrap for time series," Bernoulli 3, 123-148.

Chang, Y. and J.Y. Park (2001). "On the asymptotics of ADF tests for unit roots," mimeographed, Department of Economics, Rice University.

Datta, S. (1996). "On asymptotic properties of bootstrap for AR(1) processes," Journal of Statistical Planning and Inference 53, 361-374. 
Dickey, D. A. and W. A. Fuller (1979). "Distribution of estimators for autoregressive time series with a unit root," Journal of the American Statistical Association $74,427-431$.

Dickey, D.A. and W.A. Fuller (1981). "Likelihood ratio statistics for autoregressive time series with a unit root," Econometrica 49, 1057-1072.

Elliott, G., T.J. Rothenberg and J.H. Stock (1996). "Efficient tests for an autoregressive unit root," Econometrica, 64, 813-836.

Ferretti, N and J. Romo (1996). "Unit root bootstrap tests for AR(1) models," Biometrika 83, 849-860.

Fuller, W.A. (1996). Introduction to Statistical Time Series 2nd ed. Wiley: New York.

Kreiss, J.P. (1992). "Bootstrap procedures for AR( $\infty)$-processes," in K.H. Jöckel, G. Rothe and W. Sender (eds), Bootstrapping and Related Techniques, Lecture Notes in Economics and Mathematical Systems 376, Springer: Heidelberg.

Künsch, H.R. (1989). "The jackknife and the bootstrap for general stationary observations," Annals of Statistics, 17, 1217-1241.

Leybourne, S. and P. Newbold (1999). "On the size properties of Phillips-Perron tests," Journal of Time Series Analysis 20, 51-61.

Ng, S. and P. Perron (2000). "Lag length selection and the construction of unit root tests with good size and power," mimeographed, Department of Economics, Boston College.

Park, J. Y. (1999). "An invariance principle for sieve bootstrap in time series," mimeographed, School of Economics, Seoul National University.

Parthasarathy, K.R. (1967). Probability Measures on Metric Spaces Academic Press: New York.

Phillips, P.C.B. (1987). "Time series regression with a unit root," Econometrica 55, 277-301.

Phillips, P.C.B. and P. Perron (1988). "Testing for a unit root in time series regression," Biometrika 75, 335-346.

Said, S.E. and D.A. Dickey (1984). "Testing for unit roots in autoregressive-moving average models of unknown order," Biometrika 71, 599-608.

Stock, J. H. (1994). "Unit roots, structural breaks and trends," in R.F. Engle and D.L. McFadden (eds), Handbook of Econometrics, Vol. IV, pp. 2739-2841, Elsevier, Amsterdam. 
Table 1A: Finite sample sizes and rejection probabilities coefficient tests $S_{n}$ and $S_{n}^{*}$ for $n=50$

\begin{tabular}{|c|c|c|c|c|c|c|c|}
\hline & & $1 \%$ & test & $5 \%$ & test & $10 \%$ & test \\
\hline$\beta$ & $\alpha$ & $S_{n}$ & $S_{n}^{*}$ & $S_{n}$ & $S_{n}^{*}$ & $S_{n}$ & $S_{n}^{*}$ \\
\hline \multirow{3}{*}{-0.8} & 1.00 & 0.349 & 0.013 & 0.442 & 0.070 & 0.489 & 0.140 \\
\hline & 0.95 & 0.422 & 0.016 & 0.503 & 0.088 & 0.543 & 0.163 \\
\hline & 0.90 & 0.467 & 0.019 & 0.530 & 0.097 & 0.556 & 0.180 \\
\hline \multirow{3}{*}{-0.4} & 1.00 & 0.107 & 0.010 & 0.177 & 0.049 & 0.229 & 0.095 \\
\hline & 0.95 & 0.143 & 0.013 & 0.244 & 0.059 & 0.314 & 0.126 \\
\hline & 0.90 & 0.183 & 0.016 & 0.310 & 0.079 & 0.388 & 0.155 \\
\hline \multirow{3}{*}{0.0} & 1.00 & 0.085 & 0.007 & 0.141 & 0.039 & 0.189 & 0.081 \\
\hline & 0.95 & 0.120 & 0.009 & 0.194 & 0.057 & 0.253 & 0.112 \\
\hline & 0.90 & 0.148 & 0.013 & 0.241 & 0.074 & 0.314 & 0.143 \\
\hline \multirow{3}{*}{0.4} & 1.00 & 0.089 & 0.008 & 0.146 & 0.041 & 0.185 & 0.082 \\
\hline & 0.95 & 0.126 & 0.010 & 0.210 & 0.055 & 0.266 & 0.111 \\
\hline & 0.90 & 0.154 & 0.012 & 0.258 & 0.072 & 0.328 & 0.140 \\
\hline \multirow{3}{*}{0.8} & 1.00 & 0.115 & 0.008 & 0.167 & 0.044 & 0.211 & 0.076 \\
\hline & 0.95 & 0.152 & 0.011 & 0.229 & 0.050 & 0.280 & 0.103 \\
\hline & 0.90 & 0.189 & 0.013 & 0.271 & 0.063 & 0.321 & 0.121 \\
\hline
\end{tabular}


Table 1B: Finite sample sizes and rejection probabilities t-tests $T_{n}$ and $T_{n}^{*}$ for $n=50$

\begin{tabular}{|c|c|c|c|c|c|c|c|}
\hline & & $1 \%$ & test & $5 \%$ & test & $10 \%$ & test \\
\hline$\beta$ & $\alpha$ & $T_{n}$ & $T_{n}^{*}$ & $T_{n}$ & $T_{n}^{*}$ & $T_{n}$ & $T_{n}^{*}$ \\
\hline \multirow{3}{*}{-0.8} & 1.00 & 0.273 & 0.013 & 0.392 & 0.090 & 0.462 & 0.176 \\
\hline & 0.95 & 0.345 & 0.020 & 0.475 & 0.118 & 0.554 & 0.223 \\
\hline & 0.90 & 0.416 & 0.026 & 0.549 & 0.144 & 0.615 & 0.257 \\
\hline \multirow{3}{*}{-0.4} & 1.00 & 0.078 & 0.015 & 0.165 & 0.074 & 0.239 & 0.138 \\
\hline & 0.95 & 0.111 & 0.019 & 0.244 & 0.100 & 0.341 & 0.189 \\
\hline & 0.90 & 0.137 & 0.023 & 0.301 & 0.127 & 0.407 & 0.219 \\
\hline \multirow{3}{*}{0.0} & 1.00 & 0.073 & 0.017 & 0.154 & 0.073 & 0.224 & 0.141 \\
\hline & 0.95 & 0.100 & 0.020 & 0.220 & 0.104 & 0.305 & 0.186 \\
\hline & 0.90 & 0.114 & 0.024 & 0.246 & 0.111 & 0.340 & 0.206 \\
\hline \multirow{3}{*}{0.4} & 1.00 & 0.084 & 0.017 & 0.182 & 0.089 & 0.245 & 0.152 \\
\hline & 0.95 & 0.097 & 0.019 & 0.224 & 0.091 & 0.318 & 0.179 \\
\hline & 0.90 & 0.119 & 0.024 & 0.266 & 0.116 & 0.363 & 0.208 \\
\hline \multirow{3}{*}{0.8} & 1.00 & 0.083 & 0.013 & 0.178 & 0.073 & 0.249 & 0.143 \\
\hline & 0.95 & 0.112 & 0.019 & 0.235 & 0.101 & 0.319 & 0.185 \\
\hline & 0.90 & 0.121 & 0.023 & 0.247 & 0.105 & 0.348 & 0.197 \\
\hline
\end{tabular}


Table 2A: Finite sample sizes and rejection probabilities coefficient tests $S_{n}$ and $S_{n}^{*}$ for $n=100$

\begin{tabular}{|c|c|c|c|c|c|c|c|}
\hline & & $1 \%$ & test & $5 \%$ & test & $10 \%$ & test \\
\hline$\beta$ & $\alpha$ & $S_{n}$ & $S_{n}^{*}$ & $S_{n}$ & $S_{n}^{*}$ & $S_{n}$ & $S_{n}^{*}$ \\
\hline \multirow{3}{*}{-0.8} & 1.00 & 0.360 & 0.017 & 0.474 & 0.101 & 0.535 & 0.190 \\
\hline & 0.95 & 0.626 & 0.047 & 0.735 & 0.202 & 0.782 & 0.339 \\
\hline & 0.90 & 0.775 & 0.070 & 0.824 & 0.267 & 0.849 & 0.421 \\
\hline \multirow{3}{*}{-0.4} & 1.00 & 0.091 & 0.013 & 0.174 & 0.055 & 0.236 & 0.111 \\
\hline & 0.95 & 0.207 & 0.027 & 0.386 & 0.148 & 0.504 & 0.271 \\
\hline & 0.90 & 0.392 & 0.066 & 0.619 & 0.271 & 0.716 & 0.416 \\
\hline \multirow{3}{*}{0.0} & 1.00 & 0.069 & 0.011 & 0.129 & 0.045 & 0.188 & 0.087 \\
\hline & 0.95 & 0.152 & 0.031 & 0.300 & 0.133 & 0.412 & 0.242 \\
\hline & 0.90 & 0.270 & 0.059 & 0.491 & 0.241 & 0.627 & 0.406 \\
\hline \multirow{3}{*}{0.4} & 1.00 & 0.080 & 0.008 & 0.146 & 0.045 & 0.207 & 0.096 \\
\hline & 0.95 & 0.186 & 0.028 & 0.329 & 0.131 & 0.431 & 0.236 \\
\hline & 0.90 & 0.301 & 0.054 & 0.512 & 0.225 & 0.628 & 0.379 \\
\hline \multirow{3}{*}{0.8} & 1.00 & 0.126 & 0.010 & 0.204 & 0.048 & 0.260 & 0.091 \\
\hline & 0.95 & 0.240 & 0.023 & 0.377 & 0.107 & 0.460 & 0.202 \\
\hline & 0.90 & 0.345 & 0.035 & 0.502 & 0.163 & 0.587 & 0.285 \\
\hline
\end{tabular}


Table 2B: Finite sample sizes and rejection probabilities t-tests $T_{n}$ and $T_{n}^{*}$ for $n=100$

\begin{tabular}{|c|c|c|c|c|c|c|c|}
\hline & & $1 \%$ & test & $5 \%$ & test & $10 \%$ & test \\
\hline$\beta$ & $\alpha$ & $T_{n}$ & $T_{n}^{*}$ & $T_{n}$ & $T_{n}^{*}$ & $T_{n}$ & $T_{n}^{*}$ \\
\hline \multirow{3}{*}{-0.8} & 1.00 & 0.253 & 0.023 & 0.362 & 0.110 & 0.434 & 0.198 \\
\hline & 0.95 & 0.486 & 0.050 & 0.619 & 0.211 & 0.698 & 0.362 \\
\hline & 0.90 & 0.671 & 0.088 & 0.775 & 0.301 & 0.829 & 0.467 \\
\hline \multirow{3}{*}{-0.4} & 1.00 & 0.042 & 0.016 & 0.112 & 0.063 & 0.186 & 0.131 \\
\hline & 0.95 & 0.101 & 0.034 & 0.254 & 0.135 & 0.369 & 0.247 \\
\hline & 0.90 & 0.241 & 0.061 & 0.471 & 0.242 & 0.614 & 0.410 \\
\hline \multirow{3}{*}{0.0} & 1.00 & 0.026 & 0.015 & 0.082 & 0.062 & 0.139 & 0.110 \\
\hline & 0.95 & 0.069 & 0.033 & 0.182 & 0.116 & 0.291 & 0.216 \\
\hline & 0.90 & 0.138 & 0.056 & 0.342 & 0.211 & 0.487 & 0.358 \\
\hline \multirow{3}{*}{0.4} & 1.00 & 0.029 & 0.015 & 0.089 & 0.059 & 0.156 & 0.117 \\
\hline & 0.95 & 0.076 & 0.032 & 0.198 & 0.121 & 0.307 & 0.223 \\
\hline & 0.90 & 0.150 & 0.050 & 0.354 & 0.204 & 0.500 & 0.352 \\
\hline \multirow{3}{*}{0.8} & 1.00 & 0.031 & 0.013 & 0.091 & 0.061 & 0.153 & 0.119 \\
\hline & 0.95 & 0.068 & 0.030 & 0.189 & 0.117 & 0.285 & 0.204 \\
\hline & 0.90 & 0.114 & 0.045 & 0.283 & 0.172 & 0.414 & 0.300 \\
\hline
\end{tabular}

